\title{
Considerations in developing a scalable wind power forecasting solution
}

\author{
Shane Butler \\ Dept. of Electronic Engineering \\ Maynooth University, \\ County Kildare, Ireland. \\ Email: shanebutler20@gmail.com
}

\author{
Des Farren \\ Servusnet Informatics Ltd. \\ Co. Cork, Ireland \\ Email: desfarren@servusnet.com
}

\author{
John V. Ringwood \\ Dept. of Electronic Engineering \\ Maynooth University, \\ County Kildare, Ireland. \\ Email: john.ringwood@eeng.nuim.ie
}

\begin{abstract}
This paper addresses the wind power forecasting problem from the standpoint of performance and solution requirements. These requirements stem from the need to trade wind power on real-time energy markets, the need for reliable and accurate forecasts, the ability to learn from new data as it becomes available, the ability of the solution to generalise and scale to a large number of wind farms, and the need to compute the solutions in real time. An analogs-based solution methodology is implemented and demonstrated to show potential in fulfilling the solution requirements. Analogs-based modelling represent a non-parametric modelling solution which exploit similarities in historical data with the current forecasting window. The methodology is applied to both local wind speed forecast correction and, subsequently, to wind farm power forecasting. Results for two wind farms in Europe are presented, confirming analogs as a viable and attractive solution methodology.
\end{abstract}

\section{INTRODUCTION}

Wind energy is continuing to experience rapid growth. In the United States alone, the percentage of total electrical demand being met by wind energy tripled between 2008 and 2013 from $1.5 \%$ to $4.5 \%$ [1]. As experiences with integrating large volumes of variable wind energy production onto the electrical grid have grown, grid operators are increasingly expecting wind generators to behave like traditional power generating sources, providing forecast energy production schedules to the grid operator. Like other generating sources, wind operators are also required to cover the market costs associated with making up for differences between the energy delivery scheduled and the actual energy delivered. Additionally, wind farm operators in some markets are also being financially penalised for perceived patterns of over- or under-delivery of scheduled energy, occurring over specific intervals, or at specific times of the day [2]. Therefore, wind power forecasting represents a key technology for both grid operators - to better manage supply, and wind farm operators - to better trade their energy and avoid financial penalties for errors in scheduling.

\section{Wind POWER ForeCASTING}

In general, wind power forecasting (WPF) systems takes as input meteorological forecasts at the wind farm site and produce as output the corresponding expected wind farm power generation. Forecast values of power generation at different horizons are generally required, for different enduses. For example, energy is often traded in day-ahead markets whereas scheduling/balancing activities typically happen in the hours before delivery.

Due to the significant realisable benefits of improved wind power forecasting accuracy, it been a major focus of ongoing research in recent years. This is reflected in the diverse range of modelling approaches that have been investigated for mapping the relationship between forecast meteorological variables and wind farm power generation. A number of comprehensive reports and literature reviews are available which provide evidence for the diversity of approaches [3], [4], [5]. In addition, the IEEE Power and Energy Society (PES) has sponsored wind power forecasting competitions as part of the global energy forecasting competition GEFC, in both 2012 [6] and 2014. The best performing solutions in the 2012 competition used approaches as varied as gradientboosted decision trees [7], k-nearest neighbours algorithm [8] and ensembles of neural-networks and gaussian processes [9].

In most real-world WPF system deployments, it is typical that some period of historical wind farm observations and meteorological forecasts might be available for training and testing, typically between 6 and 24 months of data. In other deployments, for example a newly constructed wind farm, the available set of historical observations and forecasts might be minimal. Furthermore, it is highly likely that only a significant subset of all the possible meteorological conditions that a wind farm will observe during its lifetime (typically 2025 years) will occur during the period for which historical observations are available. Therefore, a key desirable feature of any WPF system is the ability to perform incremental 'learning' in response to new observations and forecasts which are continuously generated. Another desirable characteristic of a WPF solution, not frequently considered within the literature, is the ability of a solution to be 'generalised', enabling it to be scaled and deployed to potentially hundreds of wind farms with manageable implementation and maintenance overhead.

In this paper, the development of an empirical WPF system is described. In an effort to address some of the desirable WPF system characteristics described above, a case study was carried out to investigate the merits of an "analogs-based" modelling methodology to map the relationship between the relevant input and output variables in a multi-step WPF system. The layout of the remainder of this paper is as 
follows. Section III describes the design and architecture of the proposed WPF solution. Section IV then describes the analogs modelling methodology used to implement the proposed WPF solution. Section V then describes a case study, where an analogs-based WPF solution was implemented and tested on two wind farms. Some findings and results arising from this case study are also discussed. Finally, Section VI presents some conclusions.

\section{PROBLEM DESCRIPTION}

Figure 1 shows the data-flow within the WPF system. The approach involves multiple steps where the corresponding system component models a different intermediary process in mapping the relationship between forecast meteorological conditions and wind farm power generation.

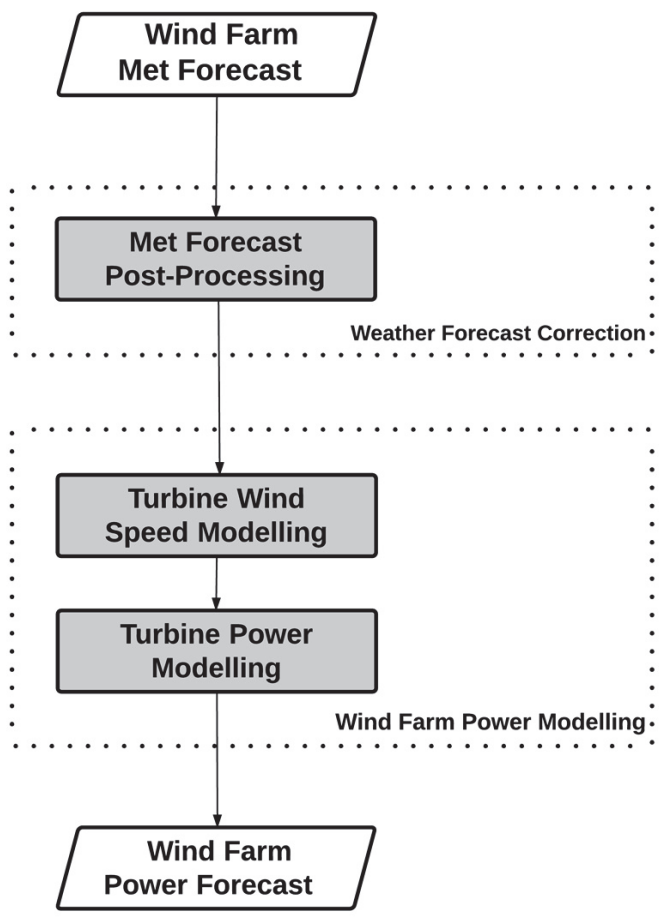

Fig. 1. Wind Power Forecasting System Flow Diagram

The first system component takes as input a site specific meteorological forecast comprising forecast values of meteorological variables at one or more location(s) in the wind farm, typically a met mast or turbine location(s). At larger wind farms, meteorological forecasts might be provided at multiple different locations in the wind farm. This system component, met forecast post-processing, seeks to make corrections to forecast values of meteorological variables to account for any systematic biases or errors in the original met forecast. The available data set for making corrections to forecast values comprises past forecast values and the corresponding observed values. For each forecast value of wind speed and wind direction in the input met forecast, a corrected value of forecast wind speed and wind direction is generated as output.

The second system component, turbine wind speed modelling, iterates through each turbine in the wind farm and, taking as input the forecast values of wind speed and wind direction at the met forecast input location(s), generates as output the corresponding forecast values of wind speed at that turbine in the wind farm. For each turbine in the wind farm, the available data set for modelling the relationship between wind speed and wind direction at the input location(s) and wind speed at the target turbine comprises all the past observations of these variables at the relevant location(s).

The final system component, turbine power modelling, takes as input the forecast values of wind speed and air density at each individual turbine, and produces as output the corresponding forecast values of power generation at each individual turbine. The available data set for modelling the relationship between input wind speed and air density and output power, at each individual turbine, comprises the historical observations of these variables at each turbine.

This approach to modeling wind farm power generation can be categorised as a disaggregated approach, where modelling of the relationship between meteorological conditions and wind farm power generation is implemented at the turbinelevel and then summed up to the wind farm level. Within the industry, it is more common for the relationship between meteorological conditions and wind farm power generation to be modeled a single step, directly mapping from meteorological conditions to total wind farm power generation. The advantage of the disaggregated approach is that it more accurately models the inter-turbine wind speed effects, resulting in more accurate modelling of the relationship between meteorological conditions and wind farm power generation. The disaggregated approach also enables a number of other useful features. In particular, by modelling at the turbine level, turbine availability and future turbine availability (e.g. scheduled maintenance) can be directly incorporated into the production of the power generation forecast. Additionally, changes in individual turbine performance characteristics (e.g. following blade upgrades) requires that updates need only be applied to the turbine power models. All the historical observations describing the interturbine wind dynamics still remain valid.

\section{Analogs Methodology for Wind Power FORECASTING}

In addition to the primary objective of seeking to maximise the power forecasting accuracy of the developed WPF system, a number of more general design considerations were made when considering the choice of data-modelling methodology. In particular, the scalability of the developed solution, its generalisation capability and the ability to perform incremental 
learning, in response to the continuous real-time stream of new forecasts and observational data becoming available, were major considerations.

To address these issues, and "analogs-based" modelling methodology is investigated and implemented to separately map the relationship between the relevant input and output variables within each WPF system component highlighted in Figure 1. The analogs-based approach was first proposed in [10] whereby different met forecast post-processing algorithms were implemented and tested. These post-processing algorithms exploit site observations to reduce any systematic biases and errors in the original forecast values of meteorological variables generated by the NWP model.

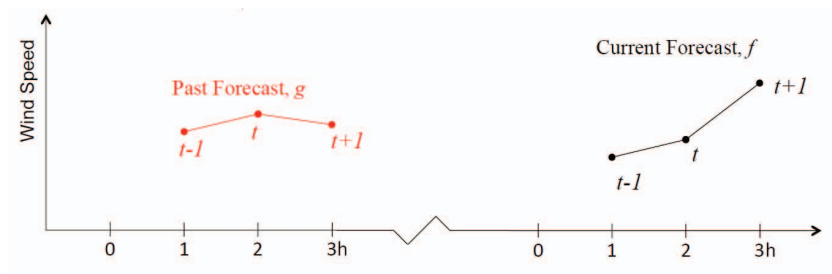

Fig. 2. The analogs scheme for met forecast post-processing [10]

In [10], the best performing algorithm identified, referred to as an "analogs scheme", involves a two-step process. Firstly, analogs of the current forecast are identified. In this case, the analog of a forecast for a given location and time is defined as a past forecast that matches selected features of the current forecast [10]. Then, once the set of the most similar past analogs are identified, according to some specified set of feature metrics defining similarity, the weighted average of the corresponding observations, for the timestamps at which the forecast analogs were valid, is computed - representing the corrected forecast value. Figure 2 illustrates the general idea behind the analogs scheme.

As identified in [10], a suitable metric for identifying past analog forecasts similar to the current forecast is as follows

$$
\left\|F_{t}, A_{t^{\prime}}\right\|=\sum_{i=1}^{N_{v}} \frac{w_{i}}{\sigma_{f_{i}}} \sqrt{\sum_{j=-\tilde{t}}^{\tilde{t}}\left(F_{i, t+j}-A_{i, t^{\prime}+j}\right)^{2}}
$$

where $F_{t}$ is the forecast to be corrected at a given time $t$ and $A_{t^{\prime}}$ is an analog forecast, which was generated in the past at time $t^{\prime}$ before $F_{t}$ was generated. $N_{v}$ and $w_{i}$ represent the number of individual forecast variables and their respective weights used in computing the forecast similarity. The variable $\sigma_{f_{i}}$ represents the standard deviation of all past forecast values of the relevant forecast variable and $\hat{t}$ represents an integer value equal to half the width of the time window over which the similarity metric is computed. $F_{i, t+j}$ and $A_{i, t^{\prime}+j}$ represent the forecast and analog values inside the time window, for the given forecast variable. The general idea of the analogs approach is to find forecasts in the past that were predicting similar values and temporal trends to the current forecast, with the assumption that if such forecast exist, their errors will likely be similar to the current forecast, according to 1 .

Once a set of analog forecasts, with respect to the current forecast, have been identified, the corrected value of the relevant forecast variable can be computed as

$$
\hat{F}_{t}=\sum_{i=1}^{N_{a}} \mu_{i} O_{i, t_{i}}
$$

where $\hat{F}_{t}$ is the corrected forecast value at time $t, N_{a}$ is the number of analogs used in the computation and $O_{i, t_{i} i=1,2, \ldots, N_{a}}$ represent the observed values of the forecast variable, corresponding to the identified forecast analogs. The weighting variable $\mu_{i}$ is computed as

$$
\mu_{i}=\frac{\frac{1}{\left\|F_{t}, A_{i, t_{i}}\right\|}}{\sum_{j=1}^{N_{a}} \frac{1}{\left\|F_{t}, A_{i, t_{i}}\right\|}}
$$

so that each analog is weighted according to the inverse distance of the analog from the current forecast. The weighting is normalised by the sum of the inverse distance computed for each analog. The result is that the observations corresponding to the most similar analogs are assigned greater weighting in computing the corrected forecast value $\hat{F}_{t}$.

In this paper, an analogs-based methodology as described above was exploited for met forecast post-processing. In addition, the principle of the analogs-based methodology was also applied for wind farm power modelling (see Figure ??). Wind farm power modelling involves both turbine wind speed modelling and turbine power modelling as described in Section ??. In applying an analogs type approach to these modelling tasks, relevant historical observations were searched to identify values similar to the latest relevant input values. The corresponding values of the output variables were then computed as the weighted sum of the past input values most similar to the current input values. For example, consider Figure 3 which illustrates a scatter plot of observed wind speed and power values from a single turbine. Each plotted value is shaded according to the corresponding observed air density value. The analogs-based methodology applied to turbine power modelling searches these historical values to identify similar pairs of wind speed and air density values, with respect to the current forecast values of wind speed and air density at this turbine. The corresponding power values of the most similar past values are used to generated a forecast value of power at that turbine.

\section{A. Discussion}

The merits of the analogs scheme are demonstrated clearly in [10], where the analogs-scheme outperformed both Kalmanfilter and time-series based approaches to correcting meteorological forecast errors. More recently, a related followup publication [11] demonstrated how the analog method is more effective at reducing random forecast errors compared to time-series or more traditional model output statistics (MOS) 


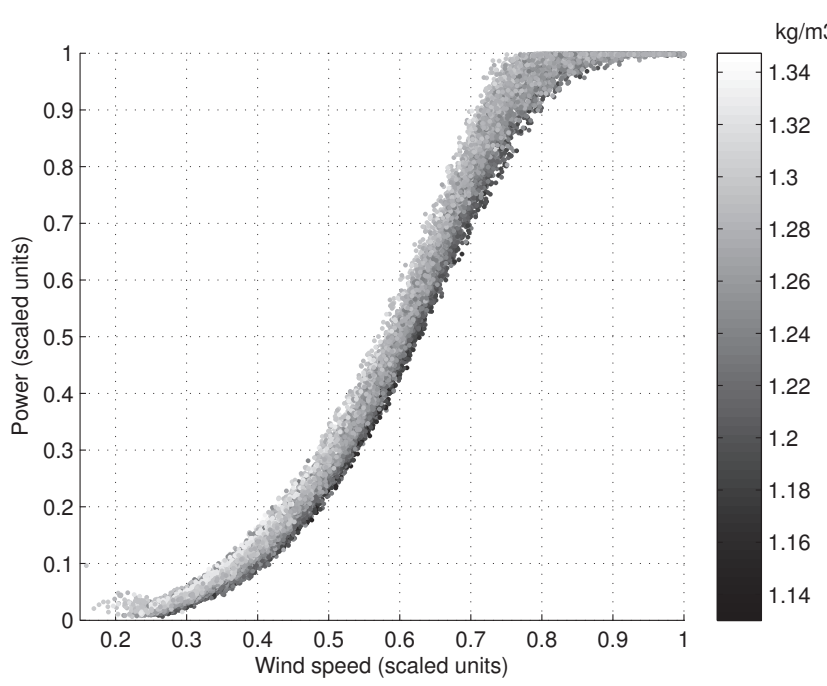

Fig. 3. Turbine power curve from operational data, showing variance due to air density variations

approaches, across a range of different NWP models and forecast variables. Further evidence supporting the merits of similarity-based modelling approaches for wind power forecasting applications was highlighted by one of the winning entries in the GEFC wind power forecasting competition 2012 which exploited a k-nearest neighbours algorithm to model wind farm power generation [9]. The analogs method has also been exploited to generate probabilistic weather predictions, called analogs ensembles, where the probability distribution of the future state of the atmosphere is estimated with a set of past observations that correspond to the best analogs of a deterministic numerical weather prediction [12].

The similarity-based modelling approach also has the potential to satisfy a number of other desirable features of a WPF system, described previously in Section II. In particular, the implementation overhead in generalising a similarity-based modelling approach to multiples of wind farms is significantly reduced, since no specific model training is required. In addition, the similarities-based modelling approach enables incremental learning since as soon as observations are recorded they are immediately added to the set of past observations available for comparison to the incoming stream of inputs.

The analogs-based modelling approach presents a number of challenges and opportunities. Specifically, choosing the values of the various parameters - for example, how many analogs to select, how to weight importance of different input variables, e.g. wind speed -v- wind direction. Recently, an investigation into 'predictor-weighting strategies' [13] identified how significant accuracy improvements were attainable by optimising the selection of predictor variables and also their weights. Though not explicitly described here, the authors have also observed how cognisance for the non-uniform distribution of forecast values and observations across the input space can lead to further accuracy improvements. For example, the majority of observations will be recorded during prevailing wind conditions and there will be far less examples of less frequently occurring conditions. Therefore, when post-processing forecast wind speed values from prevailing wind directions, it might be prudent to consider additional input variables, e.g. pressure, when comparing the similarity of the latest forecast with past observations. At less frequent wind directions, selecting a smaller number of forecast variables or number of analogs might be more appropriate.

\section{Case Study - Results}

In this paper, an analogs-based modelling methodology is applied to implement a multi-step WPF system. The analogs methodology has previously been applied to wind power forecasting [14] where, in the analogs implementation, observed wind speed values were substituted with observed power generation values to generate a power forecast. In this paper, as illustrated in Figure ??, the analogs methodology is first independently applied to met forecast post-processing. The corrected met forecast values are then separately used as inputs to the wind farm power modelling component, to generate a wind farm power forecast.

Figure 4 provides an illustrative example of the met forecast post-processing algorithm applied to make corrections to forecast wind speed values at a wind farm location in North America. The original forecast wind speed, out to a forecast horizon of approximately 4 days, was generated from a NWP model. The corrected wind speed forecast, computed by the met forecast post-processing algorithm, and the corresponding observed wind speed are also shown. Figure 4 illustrates how the original, NWP generated, wind speed forecast is negatively biased, predicting lower wind speeds than observed. It can also be observed in this example that the corrections applied to the wind speed forecast have significantly reduced the forecast bias and produced a far more accurate wind speed forecast.

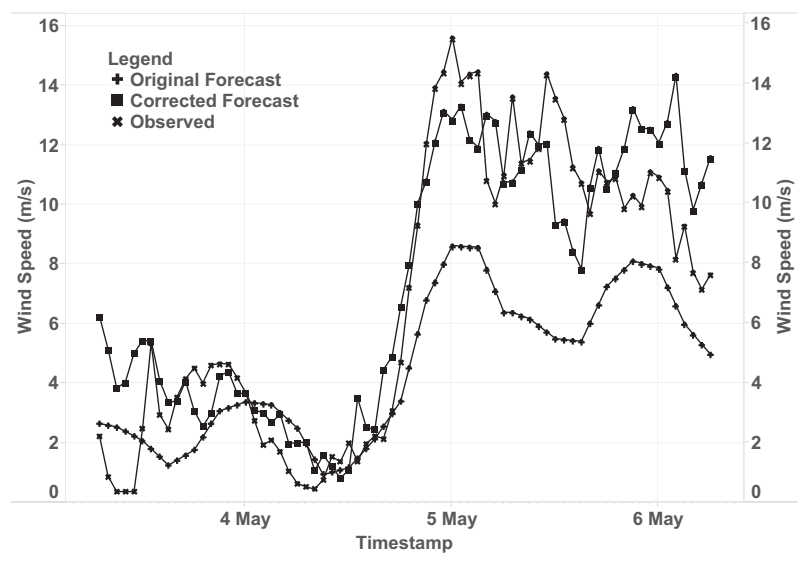

Fig. 4. Time series comparison of original wind speed forecast, corrected wind speed forecast and the corresponding observed wind speed. 
Using the original and corrected forecast meteorological variables from the example met forecast shown in Figure 4, these variables were then passed as inputs to the wind farm power modelling component to compute the corresponding power generation forecasts at the wind farm site. Figure 5 shows the corresponding power generation forecasts produced using the original and corrected forecast meteorological variables. The improvements in power forecasting accuracy when using the corrected meteorolgical forecast variables is clear. Figure also clearly 5 illustrates the non-linearity in the wind farm power modelling functionality, in terms of the relative magnitudes of the wind speed forecast errors and the resulting power forecasting errors, with respect the the original and corrected wind speed forecast values.

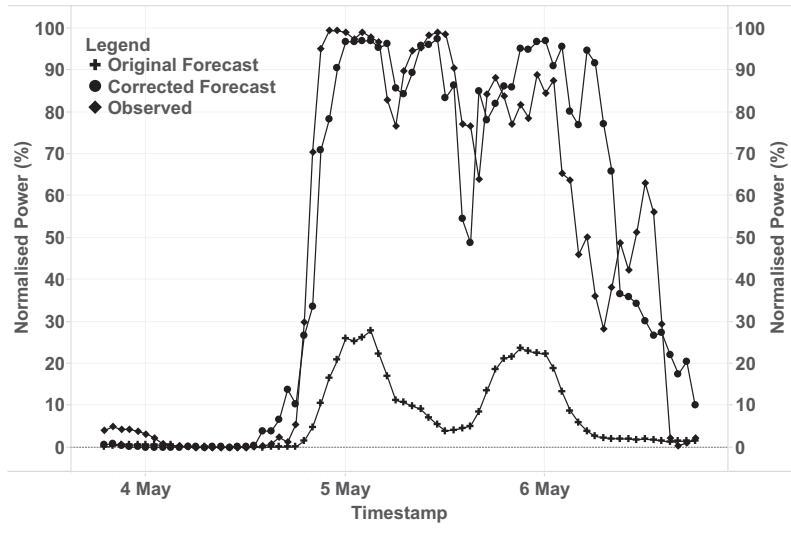

Fig. 5. Time series comparison of forecast power generation using both the original forecast meteorological variables and the post-processed/corrected forecast meteorological values. The corresponding observed wind farm power generation is also shown.

\section{A. Wind Farm Power Modelling}

To investigate the performance of the WPF system, wind power forecasts at two different wind farm locations in Europe were generated twice daily for a period of 6 months. The meteorological forecasts at the two sites were provided from an industry leading high-resolution NWP model. To investigate the added-value of the met forecasting post-processing component of the WPF system, performance metrics for both wind speed and power forecasting accuracy were generated for both sites. For both wind speed and power forecasting accuracy, the aggregated mean absolute error (MAE) was computed for each 30-minute interval in a 48 hour forecast horizon.

Figure 6 shows the computed MAE values for wind farm site 1 when comparing all the original and corrected wind speed forecast values to the observed wind speed values in 30minute intervals out to a forecast horizon of 48 hours. Figure 6 illustrates the significant improvement in wind speed forecasting accuracy arising from the met forecast post-processing algorithm.

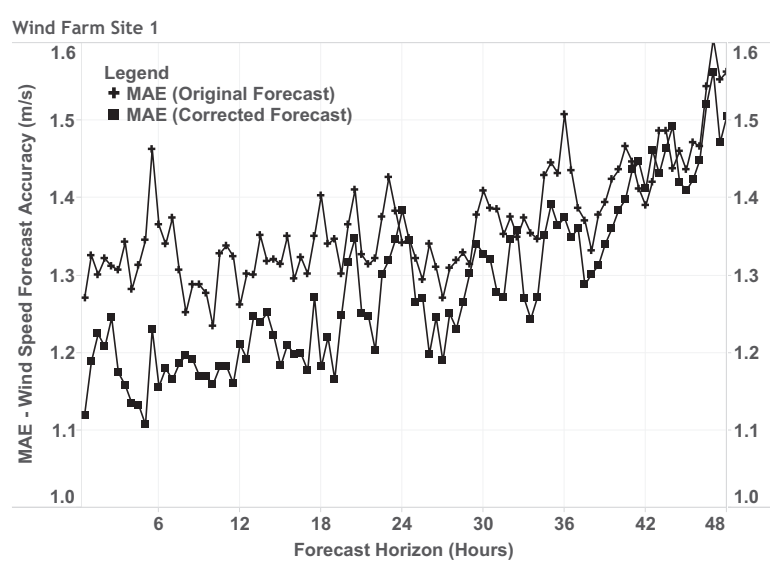

Fig. 6. Wind speed forecasting accuracy of both original and post-processed wind speed forecasts, aggregated in 30-minute intervals out to a forecast horizon of 48 hours, at wind farm site 1 .

Figure 7 compares the corresponding power forecasting accuracy when using the original and corrected meteorological forecast values, separately, as inputs to the turbine wind speed modelling component. Figure 7 clears illustrates how the improvement in wind speed forecasting accuracy shown in Figure 6 translates into similar significant improvements in power forecasting accuracy. In Figure 7, accuracy is measured using the normalised MAE (NMAE). The NMAE simply involves dividing the MAE by the maximum generating capacity of the wind farm. The NMAE enables the forecasting accuracy at wind farm sites with different generating capacities to be directly compared.

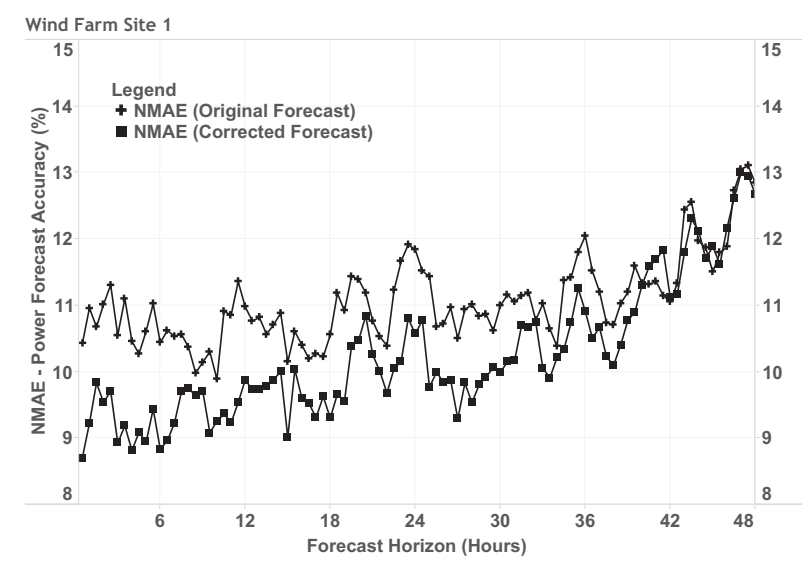

Fig. 7. Wind power forecasting accuracy of power forecasts generated using both original and post-processed wind speed forecasts, aggregated in 30minute intervals out to a forecast horizon of 48 hours at wind farm site 1 . 
Figures 8 and 9 show the corresponding wind speed and power forecasting accuracy respectively at wind farm site 2 . Most notably both the overall forecasting and accuracy and the relative improvement in accuracy arising from application of the met-forecast post-processing algorithms are reduced at wind farm site 2. Notably, the terrain at wind farm site 2 is more complex than at site 1 and also the wind profile is more volatile at site 2, which likely explains much of the difference in forecasting accuracy. However, with respect to observed industry range of standard performance accuracy values [15], the results illustrated compare favourably.

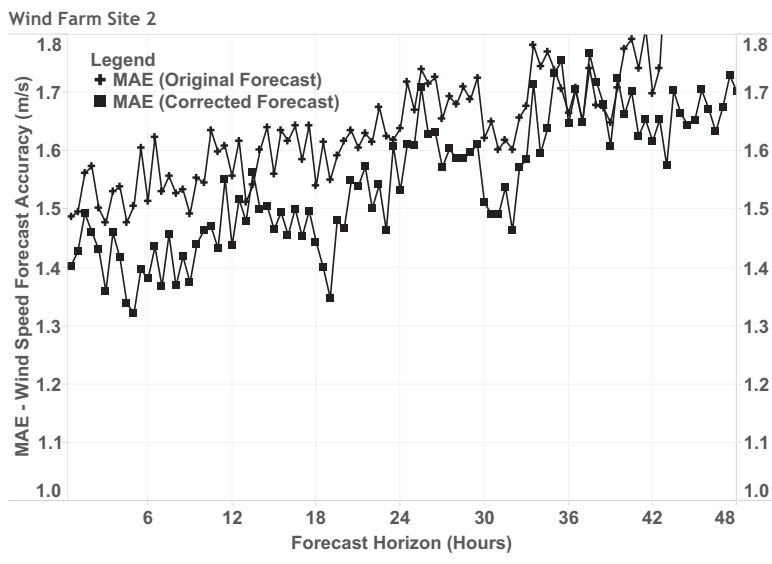

Fig. 8. Wind speed forecasting accuracy of both original and post-processed wind speed forecasts, aggregated in 30-minute intervals out to a forecast horizon of 48 hours, at wind farm site 2 .

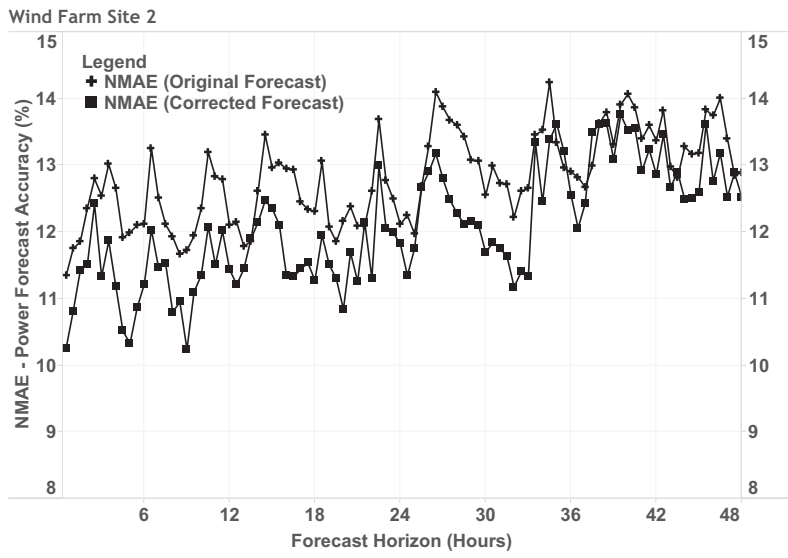

Fig. 9. Wind power forecasting accuracy of power forecasts generated using both original and post-processed wind speed forecasts, aggregated in 30minute intervals out to a forecast horizon of 48 hours at wind farm site 2 .

\section{CONCLUSION}

This paper suggests that a analogs-based machine-learning modelling approach has a number of inherent qualities which are desirable in the development of a wind power forecasting system. Specifically, the analogs-based approach is more easily scalable in that it does not require addressing issues such as model structure or model training, potentially including parameter or hyperparameter initialisation and the solving of corresponding non-convex optimisation problems. In addition, the analogs-based approach enable continuous learning without the overhead of continuous model retraining as new observations become available. This paper also illustrates how improvements in meteorological forecast accuracy, via met forecast post-processing, leads to corresponding significant improvements in power forecasting accuracy.

\section{REFERENCES}

[1] W. W. E. A. (WWEA), "Wind vision: A new era for wind power in the united states," U.S. Department of Energy, Tech. Rep., 2015. [Online]. Available: http://link.aip.org/link/?SLE/127/253/1

[2] B. P. Administration, "Generation imbalance service, version 11," Bonneville Power Administration, Tech. Rep., 2014.

[3] C. Monteiro, R. Bessa, V. Miranda, A. Botterud, J. Wang, G. Conzelmann et al., "Wind power forecasting: state-of-the-art 2009." Argonne National Laboratory (ANL), Tech. Rep., 2009.

[4] "Current methods and advances in forecasting of wind power generation,” Renewable Energy, vol. 37, no. 1, pp. 1 - 8, 2012.

[5] G. Giebel, R. Brownsword, G. Kariniotakis, M. Denhard, and C. Draxl, "The state-of-the-art in short-term prediction of wind power: A literature overview," ANEMOS. plus, Tech. Rep., 2011.

[6] T. Hong, P. Pinson, and S. Fan, "Global energy forecasting competition 2012," International Journal of Forecasting, vol. 30, no. 2, pp. 357 - 363, 2014. [Online]. Available: http://www.sciencedirect.com/science/article/pii/S0169207013000745

[7] "A feature engineering approach to wind power forecasting: \{GEFCom 2012," International Journal of Forecasting, vol. 30, no. 2, pp. 395 401, 2014.

[8] "Wind power forecasting using the -nearest neighbors algorithm," International Journal of Forecasting, vol. 30, no. 2, pp. 402 - 406, 2014

[9] D. Lee and R. Baldick, "Short-term wind power ensemble prediction based on gaussian processes and neural networks," Smart Grid, IEEE Transactions on, vol. 5, no. 1, pp. 501-510, 2014.

[10] L. Delle Monache, T. Nipen, Y. Liu, G. Roux, and R. Stull, "Kalman filter and analog schemes to postprocess numerical weather predictions," Monthly Weather Review, vol. 139, no. 11, pp. 3554-3570, 2011.

[11] B. Nagarajan, L. Delle Monache, J. P. Hacker, D. L. Rife, K. Searight, J. C. Knievel, and T. N. Nipen, "An evaluation of analog-based postprocessing methods across several variables and forecast models," Weather and Forecasting, vol. 30, no. 6, pp. 1623-1643, 2015.

[12] L. Delle Monache, F. A. Eckel, D. L. Rife, B. Nagarajan, and K. Searight, "Probabilistic weather prediction with an analog ensemble," Monthly Weather Review, vol. 141, no. 10, pp. 3498-3516, 2013.

[13] C. Junk, L. Delle Monache, S. Alessandrini, G. Cervone, and L. von Bremen, "Predictor-weighting strategies for probabilistic wind power forecasting with an analog ensemble," Meteorologische Zeitschrift, 2014.

[14] S. Alessandrini, L. Delle Monache, S. Sperati, and J. Nissen, "A novel application of an analog ensemble for short-term wind power forecasting," Renewable Energy, vol. 76, pp. 768-781, 2015.

[15] L. Landberg, J. Collings, and J. Parkes, "Taking the guesswork out of wind power forecasting," in European Wind Energy Conference (EWEA), 2012 . 